einem Tetraeder 3,$39 ; 3,73$ und $3,77 \AA$. Der kürzeste Se-Se-Abstand benachbarter Fasern beträgt 3,87 $\AA$.

Eine ausführliche Veröffentlichung ist für die Zeitschrift für anorganische Chemie vorgesehen.

Herrn Prof. Dr. U. H of $\mathrm{m}$ a $\mathrm{n} n$ und Herrn Prof. Dr. H. Witte danken wir für wertvolle Diskussionen und apparative Unterstützung.

\section{Die Auslösung der Polymerisation des Acrylamids durch Ultraschall}

Von Arnim Henglein *

Max-Planck-Institut für Chemie, Mainz

und von $\mathrm{Rolf} \mathrm{Schulz}$

Organisch-chemisches Institut der Universität Mainz

(Z. Naturforschg. 7 b, 484-485 [1952]; eingeg. am 14. Juli 1952)

Die chemischen Wirkungen von ionisierenden Strahlen und von Ultraschall in verdünnten wäßrigen Lösungen sind von mehreren Forschern untersucht und miteinander verglichen worden ${ }^{1}$. Bei den ionisierenden Strahlen besteht die Primärreaktion in einer Zersetzung des Wassers:

$$
\mathrm{H}_{2} \mathrm{O} \rightarrow \mathrm{H}+\mathrm{OH},
$$

worauf die entstandenen freien Atome und Radikale kombinieren oder auf die im Wasser gelösten Stoffe einwirken. Prudhomme und Grabar ${ }^{2}$ nehmen Gl. (1) ebenfalls als die Reaktion an, die primär in den Entladungen der Kavitationsblasen bei Durchstrahlung von gashaltigen wäßrigen Lösungen mit Ultraschall eintritt. Danach bestünden zwischen den Wirkungen des Ultraschalls und der ionisierenden Strahlen weitgehende Analogien.

Freie Radikale sind in der Lage, die Polymerisation ungesättigter organischer Verbindungen auszulösen ${ }^{3}$. Umgekehrt kann die Polymerisationsauslösung einen empfindlichen Nachweis für Radikale darstellen ${ }^{4}$. So hat D a i n t o $n^{5}$ die Existenz der durch $\gamma$-oder Röntgenstrahlen in Wasser gebildeten Radikale durch Auslösung von Polymerisationen bewiesen.

Wir haben versucht, in Analogie zu Daintons Experimenten, durch Ultraschall Polymerisationen auszulösen. Über Polymerisationen unter Einfluß des Ultraschalls ist häufig berichtet worden ${ }^{6}$; in allen diesen Fällen wurden jedoch die emulgierenden oder thermischen Wirkungen des Ultraschalls ausgenützt, während die Auslösung der Polymerisationen durch Katalysatoren erfolgte. Die depolymerisierende Wirkung des Ultraschalls ist seit langem bekannt 7, 8; da jedoch der Abbau hochpolymerer Substan-

* Ein Teil dieser Untersuchungen wurde am Institut für physikalische Chemie und Elektrochemie der T. H. Karlsruhe durchgeführt.

1 P. G ü n th e r, Angew. Chem. 60, 75 [1948]; 63, 241 [1951]; N. Mille r, Trans. Faraday Soc. 46, 546 [1950]; M. $\mathrm{H}$ a is sin s $\mathrm{k}$ u. R. O. Prudhom me, J. Chim. physique 47, 925 [1950].

2 R. O. Prudhomme u. P. Grabar, J. Chim. physique 46, 323 [1949].

3 G. V. S chulz u. G. Wittig, Naturwiss. 27, 387 [1939]. zen nicht unter einen gewissen Polymerisationsgrad führt, war prinzipiell der Aufbau aus dem Monomeren bis zu diesem Polymerisationsgrad zu erwarten.

$\mathrm{Da}$ die chemischen Wirkungen des Ultraschalls durch die Anwesenheit von Stoffen, die einen hohen Dampfdruck besitzen, unterdrückt werden 2, wurde Acrylamid als Monomeres gewählt. Acrylamid und Polyacrylamid sind in Wasser löslich, das letztere läßt sich mit Methanol ausfällen. Die Lösungen wurden unter sorgfältigem Ausschluß von Sauerstoff hergestellt. Das verwendete Wasser war in einem Strom sauerstofffreien Stickstoffs über $\mathrm{KMnO}_{4}$ und zweimal über $\mathrm{Mn}(\mathrm{OH})_{2}$ destilliert worden. In diesen Lösungen tritt bei Einwirkung von Ultraschall ohne beobachtbare Induktionsperiode Polymerisation des

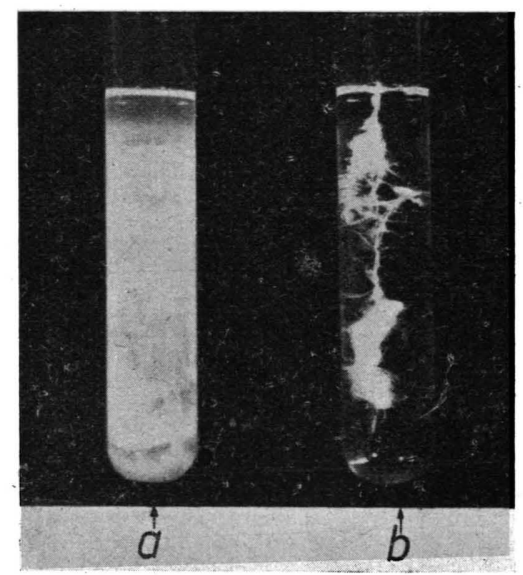

Abb. 1. Fällung des Polyacrylamids mit Methanol. a: Niedrigviskoses Produkt, entstanden durch Behandlung einer argonhaltigen 'Lösung mit Ultraschall. Es flockt gleichmäßig aus. b: Hochviskoses Produkt, entstanden durch Behandlung einer entgasten Lösung mit Ultraschall. Der Niederschlag ballt sofort zu Fäden und Klumpen zusammen. (Mittl. Molekulargewicht 4,7 Millionen).

Acrylamids ein. Ausbeute und mittleres Molekulargewicht des Polymeren hängen vom Gasgehalt der Lösung ab.

In gashaltigen Lösungen, in denen der Ultraschall depolymerisierend wirkt, entstehen Polymerisate geringen mittleren Molekulargewichtes. In argonhaltigen Lösungen entsteht ein Polymeres, das fast den gleichen Polymerisationsgrad besitzt wie das Produkt, das durch Abbau eines Hochpolymeren nach langer Schalleinwirkung ent-

4 W. Kern, Fiat review of german science. II. 5. part III, S. 196 [1948]. Makromolekulare Chem. 1, 122 [1947]; K. Z i e g l e r, Angew. Chem. 61, 177/78 [1949].

5 F. S. D a it o n, J. physic. Colloid. Chem. 52, 490 [1948].

6 A. S. Ostroski u. R. B. St a m burg h, J. appl. Physics 21, 478 [1950]; W. S a t a u. Y. H a ris a ki, Kolloid-Z. 124, 36 [1951].

7 R. O. Prudhomme u. P. Grabar, J. Chim. physique 46, 667 [1949]; R. O. Pr u d h o m m e, J. Chim. physique 47, 795 [1950]; A. W e is s l e r, J. appl. physics 21, 171 [1950].

8 G. S chmid, G. Paret u. H. Pfleiderer, Kolloid-Z. 124, 150 [1951]. 
steht. In wasserstoffhaltiger Lösung wird ein besonders hoher Umsatz an Monomerem erzielt. In Lösungen, die mit reinem Kohlendioxyd begast waren, wird keine Polymerisation beobachtet. Durch Sauerstoff oder Hydrochinon wird die Polymerisation inhibiert; dies zeigt deutlich, daß eine Radikalkettenpolymerisation vorliegt.

Werden die Lösungen durch Evakuieren entgast, tritt Polymerisation ein unter Bildung außerordentlich langer Ketten. Der Umsatz an Monomerem ist dabei kleiner als in gashaltigen Lösungen. Ein Vergleich mit anderen Wirkungen des Ultraschalls zeigt, daß die Oxydationsreaktionen in entgasten Lösungen vollständig ausbleiben, während vor kurzem eine geringe depolymerisierende Wirkung diskutiert worden ist ${ }^{8}$. Die Polymerisation als Kettenreaktion ist ein viel empfindlicheres Mittel zum Nachweis der chemischen Wirksamkeit des Ultraschalls als alle bisher beobachteten Reaktionen, wodurch verhältnismäßig große Umsätze noch unter Bedingungen erzielt werden, unter denen alle anderen Wirkungen längst ausbleiben. Wegen der stetigen Erzeugung von Radikalen in sehr geringer Konzentration erscheint es plausibel, daß in entgasten Lösungen besonders hohe Polymerisationsgrade erreicht werden (etwa 100000 ).

Die Bestimmung des mittleren Molekulargewichtes an einem dieser Polymeren geschah durch Messung der Lichtstreuung im Institut für physikalische Chemie der Universität Mainz ${ }^{9}$. Die Untersuchungen . wurden durchgeführt mit einem Magnetostriktionssender $(175 \mathrm{kHz})$ der Atlaswerke, Bremen.

Die Reaktionsgefäße wurden während der Schalleinwirkung so gut gekühlt, daß keine Temperaturerhöhung der Lösungen eintrat. Nach 1-stdg. Erhitzen einer sauerstofffreien Lösung im abgeschlossenen Gefäß auf $200^{\circ} \mathrm{C}$ war kein Polymerisat nachweisbar. Es erscheint deshalb ausgeschlossen, daß die beobachteten Polymerisationen durch die thermische Wirkung des Ultraschalls ausgelöst worden sind.

Herrn Prof. P. G ü n th er und Mitarbeitern sind wir sehr zu Dank verpflichtet für die Erlaubnis zur Benutzung des Senders und die hilfreiche Unterstützung bei den Versuchen. Herrn Prof. W. K e rn danken wir für anregende Diskussionen, Herrn Prof. F. Strass mann für das freundliche Interesse an diesen Untersuchungen.

9 Vgl. nachfolgende Mitteilung.

\section{Streulichtmessungen an wäßrigen Polyacrylsäure- amidlösungen}

Von H.-J. C a n tow

Institut für physikalische Chemie der Universität Mainz
Die Lichtzerstreuungsmessungen erfolgten in einem objektiven Streulichtphotometer. Die optische Reinigung der Lösungen geschah durch Zentrifugieren in der Meßküvette selbst bei etwa $10000 \mathrm{~g} 2$.

Das Molekulargewicht $M_{2}$ läßt sich für Moleküle, deren Größe vergleichbar mit der eingestrahlten Lichtwellenlänge ist, aus Streulichtmessungen nach folgender Formel berechnen

$$
K c_{2} / i_{\vartheta}=\left(R T / \varkappa M_{2}\right)+2 B c_{2}
$$

$i_{\vartheta} \quad$ Intensität derStreustrahlung im Einheitsvolumen im Einheitsabstand bei derWellenlänge $\lambda$ unter $\operatorname{dem}$ Winkel $\vartheta$ für die Primärstrahlintensität 1

$$
\left[\mathrm{cm}^{-1}\right]
$$

* $\quad$ Schwächungsfaktor für innermolekulareInterferenz

B 2. Virialkoeffizient des os-

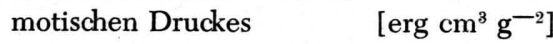

$c_{2} \quad$ Konzentration des Polymeren im Lösungsmittel $\left[\mathrm{g} \mathrm{cm}^{-3}\right]$

$R \quad$ Allgemeine Gaskonstante $\quad\left[\operatorname{erg} \operatorname{grad}^{-1} \mathrm{~mol}^{-1}\right]$ $T \quad$ Absolute Temperatur [grad]

$$
K=2 \pi^{2} n^{2}{ }_{1} R T\left(n-n_{1} / c_{2}\right)^{2} / \lambda^{4} N_{\mathrm{L}}
$$

$n_{1} \quad$ Brechungsindex des Lösungsmittels

n Brechungsindex der Lösung $N_{\mathrm{L}} \quad$ Loschmidtsche $\mathrm{Zahl} \quad\left[\mathrm{mol}^{-1}\right]$

$n-n_{1} / c_{2}$ Brechungsinkrement des Polymeren im Lösungśmittel $\left[\mathrm{cm}^{3} \mathrm{~g}^{-1}\right]$

Der Schwächungsfaktor $\varkappa$ ist von Molekülgestalt und -größe abhängig. Da bisher nur ein Polyacrylamid untersucht wurde, kann noch nicht mit Sicherheit für dies Polymere ein bestimmtes Molekülmodell angenommen werden: Wahrscheinlich kommt seine Molekülkonfiguration dem Modell der statistisch geknäuelten Fadenkette nach den Vorstellungen von $\mathrm{Kuh}^{3}$ nahe. Sicher befinden sich die Polyacrylamidmoleküle nicht als stark anisotrope Teilchen in Lösung, da das unter $90^{\circ}$ abgestrahlte Licht kaum depolarisiert ist. Für statistisch geknäuelte Fadenketten sind zwei Streufunktionen berechnet worden. Davon gilt eine für einheitlich lange Ketten, die andere für solche, deren Längenverteilung der Funktion $f\left(M_{2}\right)$ $=a M_{2} e^{-b M_{2}}$ folgt. Letztere Funktion ist für unfraktionierte Polymere anwendbar. Wir setzen sie daher im vorliegenden Falle an. Die von $\mathrm{Z}$ i m m ${ }^{4}$ abgeleitete Funktion für den Schwächungsfaktor lautet:

$$
\begin{gathered}
x=1-x /(2+x) \\
x=\left(8 \pi^{2} n^{2}{ }_{1} d^{2} \text { st } \sin ^{2} \vartheta / 2\right) / 3 \hat{\lambda}^{2} .
\end{gathered}
$$

2 H.-J. C a n t o w, erscheint demnächst in dieser Zeitschrift.

3 W. K u h n, Kolloid-Z. 68, 2 [1934].

4 B. H. Z i m m, J. chem. Physics 16, 1093, 1099 [1948].
1 Hierüber ist in der vorangehenden. Mitteilung berichtet.
$\mathrm{Hengle}$ in und $\mathrm{Schulz}^{1}$ in entgaster wäßriger Lösung aus dem Monomeren unter dem Einfluß von Ultraschallwellen hergestellt war. 